\title{
Multiscale Considerations in Modeling of Nonlinear Elastomers
}

\author{
H.T. Banks ${ }^{123}$ and Negash G. Medhin ${ }^{4}$ \\ Center for Research in Scientific Computation \\ North Carolina State University \\ Raleigh, NC, 27695-8205 \\ Gabriella A. Pinter ${ }^{5}$ \\ Department of Mathematical Sciences \\ University of Wisconsin, Milwaukee \\ Milwaukee, WI, 53201-0413
}

\begin{abstract}
We present a survey of results from an extended project focused on the understanding of the dynamic behavior of elastomers or filled rubbers. This entailed experimental, modeling, computational and theoretical efforts. Of particular emphasis are the nonlinear and hysteretic aspects of dynamic deformations.
\end{abstract}

Keywords: nonlinear partial differential equations, computational methods, viscoelasticity, hysteresis, molecular dynamics, multiscale modeling

Mathematics Subject Classification: 35L70, 74S05, 34C55, 74A99, 93A30

\section{Introduction}

In this presentation we summarize joint research efforts between scientists at the Thomas Lord Research Center of the Lord Corporation and applied mathematicians in the Center for Research in Scientific Computation (CRSC) at N.C. State University beginning in 1994 and continuing through the current efforts by the authors of this report. The research participants from Lord were Lynn Yanyo, Mike Gaitens, Beth Muñoz, and Oon Hock Yeoh, while significant CRSC contributors include H.T. Banks (1994- ), Yue Zhang (1994-1997), Nancy Lybeck (1995-1997), Laura Potter (1996-1998), Gabriella Pinter (1997- ) and Negash Medhin (2000- ). As should be apparent from our outline below (presented in a somewhat

\footnotetext{
${ }^{1}$ htbanks@eos.ncsu.edu

${ }^{2}$ Corresponding author

${ }^{3}$ Plenary Lecture, The 47th European Study Group with Industry and the Mathematics for Industry Workshop, August 24-29, 2003, Graasten, Denmark.

${ }^{4}$ ngmedhin@eos.ncsu.edu

${ }^{5}$ gapinter@uwm.edu
} 
chronological format), this project is a prime example of one wherein a data-driven application subsequently leads to new computational, theoretical and conceptual ideas. It results in a multiscale framework that connects pseudo-phenomenological or phenomenological approaches to molecular considerations. Our focus in these efforts has been the understanding of nonlinearity and hysteresis and the roles they play in the dynamic deformations of filled rubber.

An important area where nonlinear models have a potential impact is the rubber industry. Elastomeric materials can be found in diverse engineering applications, e.g., in springs, bearings, shock absorbing bushes, helicopter rotor suspensions, tires, vibration suppression devices, bridge supports. They appear both as passive damping devices and actively controllable smart materials. These new applications motivate the need for a better understanding of the mechanical behavior of rubber-like composites which is a necessary first step in the design of both passive and active material devices $[1,2]$. The dynamic behavior of these elastomers is very complex. They exhibit significant nonlinearities in both geometric and material characteristics. Typical nonlinear behaviors of the stress and strain in rubber materials under finite deformation include a continuous increase of strain at decreasing rates upon loading, variable magnitudes of strain subject to rates of loading and different loading and unloading paths due to hysteretic memory effects. In addition, the current state of material depends on the strain/strain-rate history, the type and amount of filler in the material and the temperature. The nonlinear effects are particularly strong for large strains and for highly-filled elastomers. We have attempted to accurately capture the nonlinear dynamic as well as hysteretic effects which we discuss briefly next.

Hysteresis is a widespread phenomenon in science and engineering. It plays significant roles in electromagnetics (polarization, conductivity) in dielectric and conductive materials, in biological systems (time delays in disease pathogenesis, intracellular metabolic pathways, tissue viscoelasticity), in recent investigations of ecological migrations (diffusion with integro-differential terms representing local episodic behavior) as well as in the deformations of polymeric materials such as filled rubbers. Hysteresis, often referred to as "memory", does not involve physical memory mechanisms, but rather is a manifestation of "hidden" or unmodeled internal or local dynamics. It is an embodiment of the multiscale aspects of models and phenomena. That is, one encounters Preisach to Boltzmann to internal dynamics to molecular-based models as one moves from macro to micro to nano and molecular formulations. Thus, approaches range from the phenomenological involving an input/output viewpoint to a pseudo-phenomenological involving internal dynamics formulations of Boltzmann hysteresis operators to physics-based models (e.g., the stick-slip models of Doi/Edwards and Johnson/Stacer that are discussed below) at the molecular level for internal dynamics.

\section{Models for elastomeric materials}

Traditionally there are two approaches to modeling rubber materials. There are physics or molecular-based theories $[3,4,5]$ that try to describe the microscopic behavior of the particles and fibers that constitute the material and there are phenomenological models that treat 
the material as a continuum $[6,7,8,9,10,11]$. Phenomenological models are based on the assumption that the material is isotropic, i.e., the long chain molecules are randomly oriented in the unstrained state. Loading causes orientation of the molecules, but it is in the direction of the loading, so the assumption of isotropy remains valid. Most such models utilize a strain energy density to describe the state of the material. However, these strain-energy functions can typically capture only the current state of the rubber and cannot distinguish between different loading and unloading histories. Thus these models cannot accurately describe the significant hysteresis exhibited by filled rubber samples.

\section{$2.1 \quad$ Model development}

In developing a model for the dynamic behavior of filled elastomers we considered two basic deformations: extension and shear. Our hysteretic models were based on the basic models developed by Banks et. al. in $[12,13]$. First we considered the model of a rubber rod under uniaxial tension. (Our shear results are summarized in Section 2.4.) The Timoshenko theory for longitudinal vibrations of a rubber bar with a tip mass leads to

$$
\begin{aligned}
& \rho A_{c} \frac{\partial^{2} u}{\partial t^{2}}-\frac{\partial}{\partial x}\left(A_{c} g_{e}\left(\frac{\partial u}{\partial x}\right)+A_{c} C_{D} \frac{\partial^{2} u}{\partial t \partial x}\right)=0 \quad 0<x<\ell \\
& M \frac{\partial^{2} u}{\partial t^{2}}(t, \ell)=-\left.\left(A_{c} g_{e}\left(\frac{\partial u}{\partial x}\right)+A_{c} C_{D} \frac{\partial^{2} u}{\partial t \partial x}\right)\right|_{x=\ell}+f(t)+M g \\
& u(t, 0)=0, \quad u(0, x)=\varphi_{0}(x), \quad \frac{\partial u}{\partial t}(0, x)=0,
\end{aligned}
$$

where $u$ is the deformation in the $\mathrm{x}$ direction, $\rho$ is the mass density, $f(t)$ is the applied external force, $A_{c}$ is the cross-sectional area, $M$ is the tip mass and $g$ is the gravitational constant. This model includes a Kelvin-Voigt type term as a first approximation to damping. The stress-strain relationship in the basic model is

$$
\sigma(t)=G(\tilde{e}(t), \dot{\tilde{e}}(t))
$$

where $\tilde{e}$ is the finite strain (since we are interested in large deformations) and it is given by

$$
\tilde{e}=\frac{\partial u}{\partial x}+\frac{1}{2}\left(\frac{\partial u}{\partial x}\right)^{2}=\varepsilon+\frac{1}{2} \varepsilon^{2}
$$

where $\varepsilon=\frac{\partial u}{\partial x}$ is the usual infinitesimal strain of linear elasticity. However, modeling the nonlinear behavior between the stress and the finite strains $\tilde{e}$ (which are themselves nonlinear functions of the infinitesimal strains $\varepsilon$ ) can be equivalently formulated in terms of nonlinear relationships between the stress and the infinitesimal strains $\varepsilon$. Hence, we have equivalently assumed in obtaining (1) a stress-strain relationship

$$
\sigma(t)=\tilde{g}_{e}\left(\frac{\partial u}{\partial x}, \frac{\partial^{2} u}{\partial t \partial x}\right)=g_{e}\left(\frac{\partial u}{\partial x}\right)+C_{D} \frac{\partial^{2} u}{\partial t \partial x}
$$




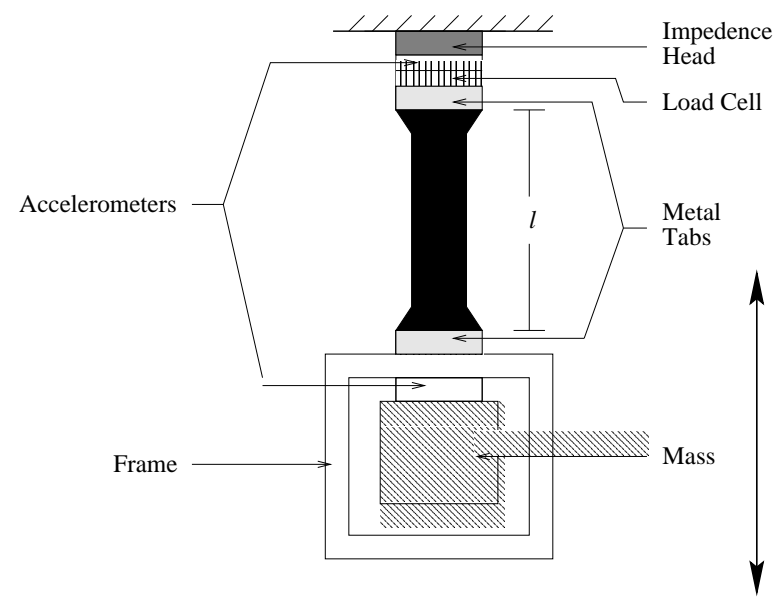

Figure 1: Elastomer rod under tension for dynamic free release experiments.

instead of a similar law involving the finite strains $\tilde{e}$.

The well-posedness of the initial-boundary value problem associated with (1)-(3) was shown by Banks, Gilliam, Shubov in [14], while the convergence properties of the corresponding parameter estimation problem were established by Banks, Pinter in [15]. These results are valid for a broad class of nonlinearities. This is important since comparison between experimental data and numerical calculations suggested that the neo-Hookean nonlinearity (found in literature as a first approximation to the nonlinearity exhibited by rubber materials) is not adequate to describe the behavior of filled elastomers [13].

An adequate form of $g_{e}$ and the unknown constants $\rho$ and $C_{D}$ were determined using parameter estimation techniques. Data for the inverse problem were provided by dynamic free release experiments. The elastomer was suspended vertically with the top end $(x=0)$ fixed, and a frame was attached to the lower end (see Figure 1). Varying amounts of extra mass were attached to this frame, which also served to house an accelerometer. Another accelerometer placed at the top of the sample was used to verify the clamped boundary condition at the fixed end. For the free release experiment, the rubber rod was lifted together with the frame and the tip mass so that no compression or extension occurred. Then the support was removed, allowing the mass to fall freely. This type of experiment was repeated with unfilled and lightly filled carbon black samples, while a similar experiment was done with a highly filled sample with a $9.29 \mathrm{lb}$ tip mass.

The force data collected by a load cell on top of the sample were used in estimating the unknown parameters $q=\left(g_{e}, \rho, C_{D}\right)$ in (1)-(3) by minimizing

$$
J(q)=\frac{1}{2} \sum_{i=1}^{N}\left|z_{i}-A_{c} \sigma\left(\bar{t}_{i}, 0 ; q\right)\right|^{2}
$$

over $q$ in some admissible parameter space $Q$. Here, $z_{i}, i=1 \ldots N$, represent the experimental observations at times $\bar{t}_{i}$ of the force at the fixed end, and $\sigma$ is given by (4), where $u$ is the solution of (1)-(3) corresponding to the parameters $q$. 
Computational results indicated that (i) a nonlinear function $g_{e}$ is necessary in the stressstrain relationship, (ii) the stress-strain relationship (4) is adequate to describe unfilled rubber samples (Figure 2) and (iii) to capture the dynamics of filled elastomers, hysteresis must be taken into account. Based on models for hysteretic damping in the literature (see
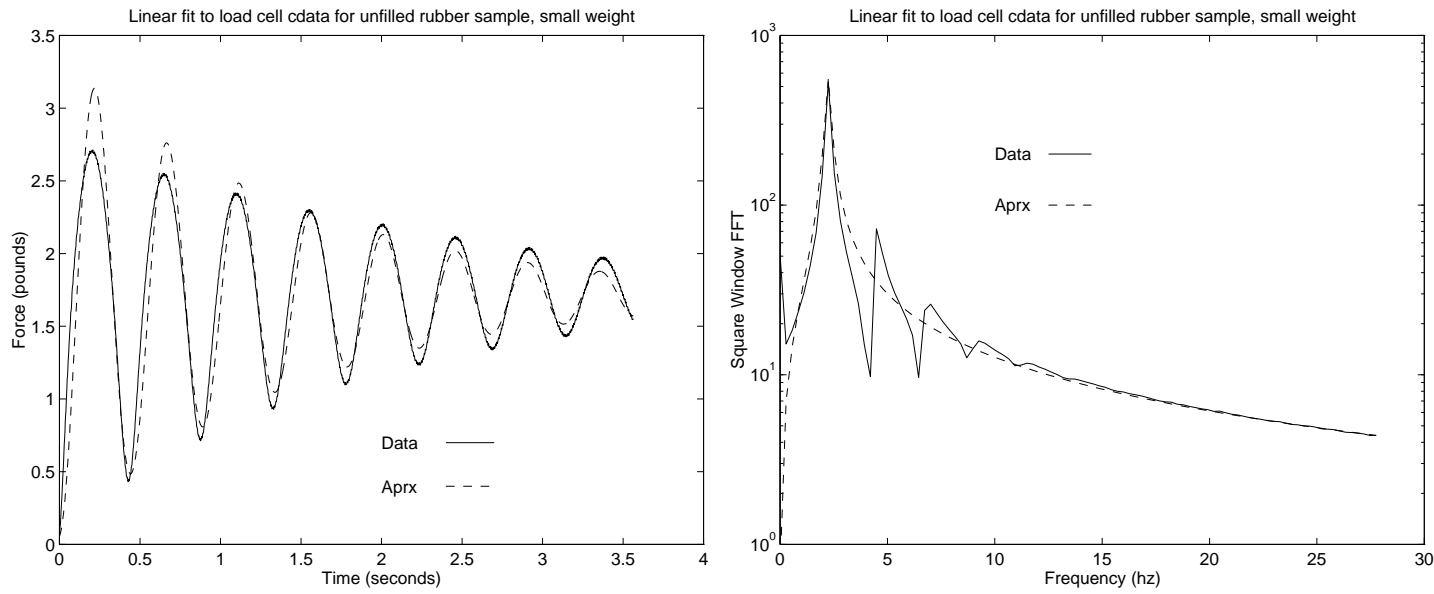

Figure 2: (a) Time domain approximation and (b) the FFT of the solution vs. the data: Model with a four-term piecewise linear $g_{e}$.

[16] and references therein) we assumed a Boltzmann-type stress-strain law of the form

$$
\sigma(t)=g_{e}(\varepsilon(t))+C_{D} \dot{\varepsilon}(t)+\int_{-\infty}^{t} Y(t-s) \frac{d}{d s} g_{v}(\varepsilon(s), \dot{\varepsilon}(s)) d s
$$

where $Y$ is the memory kernel, and $g_{e}$ and $g_{v}$ are nonlinear functions accounting for the elastic and viscoelastic nonlinear responses of the elastomer, respectively. This stress-strain law implies that the stress depends not only on the current strain and strain rate but also on the history of the strain and the strain-rate. It is very important to note that the stress-strain law (5) contains various standard internal strain or internal variable formulations as special cases. The anelastic displacement field (ADF) models of Lesieutre [17, 18] for composite materials exhibiting both elastic and anelastic displacement fields are formulated on the assumption that the host elastic material contains anelastic materials with internal strains $\varepsilon_{1}$ which are elastic strain driven. That is, the constitutive laws have the form

$$
\sigma(t)=E_{1} \varepsilon(t)-E_{2} \varepsilon_{1}(t)
$$

where the internal strain is given by

$$
\dot{\varepsilon}_{1}(t)+\frac{1}{\tau} \varepsilon_{1}(t)=c_{2} \varepsilon(t), \quad \varepsilon_{1}(0)=0
$$

or equivalently,

$$
\varepsilon_{1}(t)=\int_{0}^{t} c_{2} e^{-\frac{t-s}{\tau}} \varepsilon(s) d s
$$


Several generalizations of this formulation exist, e.g., Johnson et.al. [19], suggest that the internal strain is strain rate driven, i.e.,

$$
\dot{\varepsilon}_{1}(t)+\frac{1}{\tau} \varepsilon_{1}(t)=c_{2} \dot{\varepsilon}(t)
$$

Our Boltzmann-type law (5) (under appropriate assumptions on the past memory from $-\infty$ to 0 ) corresponds to an internal strain model of the form

$$
\dot{\varepsilon}_{1}(t)+\frac{1}{\tau} \varepsilon_{1}(t)=\frac{d}{d t} g_{v}(\varepsilon(t), \dot{\varepsilon}(t)), \quad \varepsilon_{1}(0)=0 .
$$

This form was chosen after we found that neither (6) nor (7) provided laws that could describe our data.

Experimental observations of the quasi-static behavior of elastomers indicate that these materials possess different nonlinear viscoelastic responses in loading $(\dot{\varepsilon}>0)$ and unloading $(\dot{\varepsilon}<0)$. This led to our choice of a piecewise continuous form for the viscoelastic response function $g_{v}$

$$
g_{v}(\varepsilon(s), \dot{\varepsilon}(s))= \begin{cases}g_{v i}(\varepsilon(s)) & \dot{\varepsilon}(s)>0 \\ g_{v d}(\varepsilon(s)) & \dot{\varepsilon}(s)<0\end{cases}
$$

Here we require $g_{v i}$ and $g_{v d}$ to be continuous (and generally nonlinear) functions. The difference between loading and unloading is more pronounced as the amount of filler increases in the material. We define points $t_{i}, i=1, \ldots, M$, as the "turning points," or the points in time for which $\dot{\varepsilon}=0$. The function $g_{v}$ need not be continuous at the turning points, so we must interpret the derivatives in (5),(8) as distributional derivatives. That is, delta functions are involved in differentiating the composite functions $g_{v}(\varepsilon, \dot{\varepsilon})$, or equivalently, integration by parts is valid. For experimental and computational purposes, we further assume that any motion in the material prior to each experiment is negligible. That is, we assume that $\frac{d}{d t} g_{v}(\varepsilon(t), \dot{\varepsilon}(t)) \approx 0$ for all $t<0$. Hence we may approximate $(5)$ by

$$
\sigma(t)=g_{e}(\varepsilon(t))+C_{D} \dot{\varepsilon}(t)+\int_{0}^{t} Y(t-s) \frac{d}{d s} g_{v}(\varepsilon(s), \dot{\varepsilon}(s)) d s .
$$

We integrated by parts in (10) and obtained the model in variational form

$$
\begin{aligned}
& \tilde{\rho} \frac{\partial^{2} u}{\partial t^{2}}-A_{c} \frac{\partial}{\partial x}\left(g_{e}\left(\frac{\partial u}{\partial x}\right)+Y(0) g_{v}\left(\frac{\partial u}{\partial x}, \frac{\partial^{2} u}{\partial t \partial x}\right)+\int_{0}^{t} \dot{Y}(t-s) g_{v}\left(\frac{\partial u}{\partial x}(s), \frac{\partial^{2} u}{\partial t \partial x}(s)\right) d s\right. \\
& \left.+C_{D} \frac{\partial^{2} u}{\partial t \partial x}+\sum_{k=1}^{K} Y\left(t-t_{k}\right)(-1)^{k+1}\left[g_{v i}\left(\frac{\partial u}{\partial x}\left(t_{k}\right)\right)-g_{v d}\left(\frac{\partial u}{\partial x}\left(t_{k}\right)\right)\right]\right)=F(t) \text { in } V^{*} \\
& u(t, 0)=0, \quad u(0, x)=\varphi_{0}, \quad \frac{\partial u}{\partial t}(0, x)=0
\end{aligned}
$$

for an appropriately chosen Hilbert space $V$. This presumes, of course, that we have sufficient smoothness so that evaluation of $\frac{\partial u}{\partial x}$ at $t_{i}$ makes sense and $\frac{\partial}{\partial x}\left(g_{v i}\left(\frac{\partial u}{\partial x}\left(t_{i}\right)\right)\right), \frac{\partial}{\partial x}\left(g_{v d}\left(\frac{\partial u}{\partial x}\left(t_{i}\right)\right)\right) \in$ 
$V^{*}$. For this particular example, one takes $V=H_{L}^{1}(0, \ell)=\left\{\phi \in L^{2}(0, \ell) \mid \phi^{\prime} \in L^{2}(0, \ell), \phi(0)=\right.$ $0\}$ and $\tilde{\rho}=\rho A_{c}-M \delta_{\ell}, \quad F(t)=-f(t) \delta_{\ell}$, where $\delta_{\ell}(x)$ is the Dirac function with atom at $x=\ell$. The important question of well-posedness of the system (11)-(12) is treated carefully in [20]. We have shown that under certain assumptions there exists a unique weak solution of the system (11)-(12). This result uses the Minty-Browder monotonicity method to treat the nonlinearities in the system and is valid for a broad range of elastic and viscoelastic response functions.

\subsection{Experimental and computational results}

We first tested the hysteretic model (11)-(12) in the quasi-static behavior of filled rubber samples under uniaxial tension. Two different types of quasi-static pull tests were conducted. The first type (Type I) includes a sequence of loading and unloading the sample to produce load-displacement curves with decreasing maximum strain levels. In the second type of experiment (Type II) we created a sequence of strain loops that have decreasing maximum strain levels as before, but instead of a fixed minimum strain level we used progressively increasing minimum strain levels. (Full description of the quasi-static and dynamic tests and results are given in our paper [21].) We tried a number of linear and nonlinear functions for $g_{e}$ and $g_{v}$, including the special cases of $g_{e}$ and $g_{v}$ linear with $g_{v} \equiv g_{v i} \equiv g_{v d}$. The relative errors discussed in [22] suggested that nonlinear functions are necessary for both $g_{e}$ and $g_{v}$. Additional curve fitting studies outlined in [22] led to our choice of cubic polynomials for $g_{e}, g_{v i}$ and $g_{v d}$. That is, we chose parameterized nonlinearities of the form $g_{e}(x)=\sum_{i=1}^{3} E_{i} x^{i}, \quad g_{v i}(x)=\sum_{i=1}^{3} a_{i} x^{i}, \quad g_{v d}(x)=\sum_{i=1}^{3} b_{i} x^{i}$, where $E_{i}, a_{i}, b_{i}, i=1,2,3$ are real constants. Note that these response functions include no constant terms. Here we require $g_{e}(0)=g_{v i}(0)=g_{v d}(0)=0$ so that a zero strain will yield a zero stress according to our stress-strain relation. Based on additional studies detailed in [22], we chose an exponential form $Y(s)=e^{-\frac{s}{\tau}}$ for the memory kernel. Such an exponential form generates totally nested hysteresis loops in the stress-strain curves, a feature also observed in our data.

The parameters $\tau, E_{i}, a_{i}$ and $b_{i}$ were estimated by setting up a least squares minimization problem using one or two of the outer stress strain data loops from the experiments. Figure 3 shows results for a highly filled sample in Type I and Type II experiments, respectively. While the inverse problem was "trained" on the largest loop only in Type I, and the two outer loops in the Type II experiment, we can see that there is a very good agreement between the data and the model predicted inside loops. Similar results were obtained for medium-filled natural rubber and silica-filled silicon samples. More details are given in [21].

Our hysteretic model (11)-(12) was also tested on a series of dynamical experiments with different types of filled rubber samples. We used the same free release experiments that were described earlier. Since the dynamical behavior of the unfilled natural rubber sample was adequately described by the basic model without hysteresis, we began our hysteresis investigations using the lightly filled sample. For given $\rho, C_{D}, g_{e}, g_{v}$ and $Y$ we solve the partial differential equation (11)-(12) forward in time, and obtain the displacement $u(t, x), 0 \leq x \leq$ $\ell$. The data collected in these experiments provides us with the force at the top of the sample 

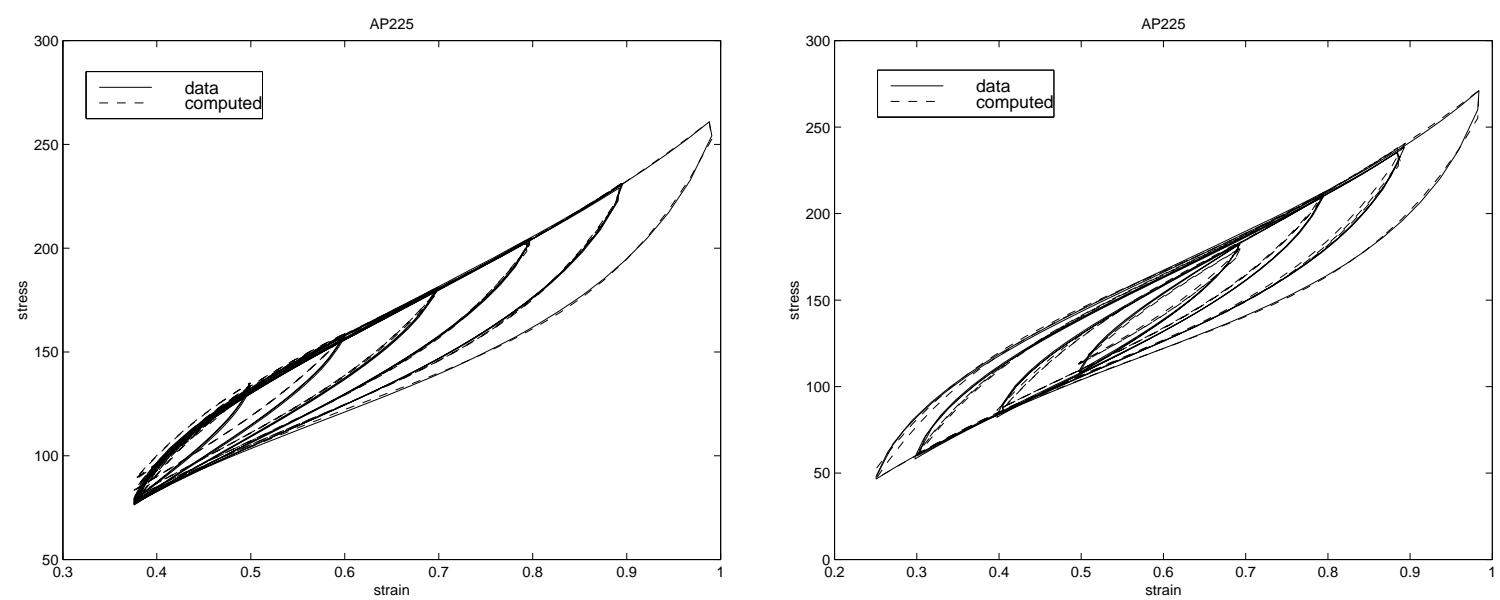

Figure 3: (a) Model prediction for Type I CB-h data and (b) Model prediction for Type II CB-h data.

$(x=0)$, and we compare it to the force predicted by the model at the same point, given by

$$
A_{c} \sigma(t, 0)=A_{c}\left[C_{D} \frac{\partial^{2} u}{\partial t \partial x}(t, 0)+g_{e}\left(\frac{\partial u}{\partial x}(t, 0)\right)+\int_{0}^{t} Y(t-s) \frac{d}{d s} g_{v}\left(\frac{\partial u}{\partial x}(s, 0), \frac{\partial^{2} u}{\partial t \partial x}(s, 0)\right) d s\right]
$$

where we use our computed solution $u(t, x)$ to find $\frac{\partial u}{\partial x}(t, 0)$ and $\frac{\partial^{2} u}{\partial t \partial x}(t, 0)$. Our goal is to find $\rho, C_{D}, g_{e}, g_{v}$ and $Y$ so that the model predicted force at $x=0$ best matches the data collected by the load cell. A parameter identification problem was set up to find $\rho, C_{D}, E_{1}, E_{2}, E_{3}, a_{1}, a_{2}, a_{3}, b_{1}, b_{2}, b_{3}, \tau$ (collectively denoted by $q$ ) such that

$$
J(q)=\sum_{i=1}^{N}\left|z_{i}-A_{c} \sigma\left(\bar{t}_{i}, 0 ; q\right)\right|^{2}
$$

is minimized. Here, once again, the $z_{i}, i=1, \ldots, N$, are the data collected by the load cell, and $\sigma\left(\bar{t}_{i}, 0 ; q\right)$ is given as described above. The particular forms for $g_{e}, g_{v}$ and $Y$ were motivated by their success in the quasi-static case. In our computations we used linear splines for spatial discretization. In solving the system (11)-(12) forward in time, the treatment of the hysteresis integral proved to be very time consuming. Since this computation needs to be repeated for each set of parameters during the optimization, the time required for the computational parameter identification process was very extensive. Hence, we formulated an equivalent system to (11)-(12) using an internal variable $\varepsilon_{1}=\varepsilon_{1}(t ; \tau)$, and used it in the above framework for our subsequent calculations. This system is given by

$$
\begin{aligned}
& \tilde{\rho} \frac{\partial^{2} u}{\partial t^{2}}-A_{c} C_{D} \frac{\partial^{3} u}{\partial t \partial x^{2}}-\frac{\partial}{\partial x}\left(A_{c} g_{e}\left(\frac{\partial u}{\partial x}\right)+A_{c} \varepsilon_{1}\right)=F(t) \text { in } V^{*} \\
& \dot{\varepsilon}_{1}=-\frac{1}{\tau} \varepsilon_{1}+\frac{d}{d t} g_{v}\left(\frac{\partial u}{\partial x}, \frac{\partial^{2} u}{\partial t \partial x}\right)
\end{aligned}
$$



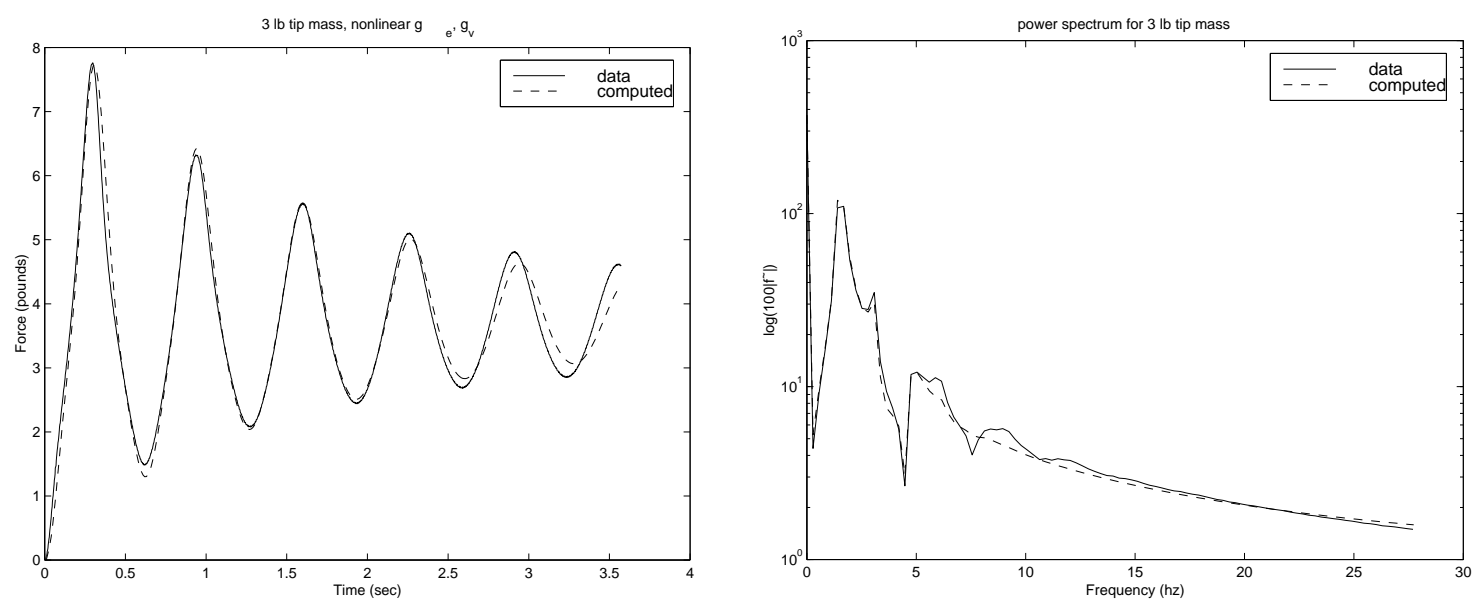

Figure 4: Fit to data with model using a nonlinear $g_{e}, g_{v}:$ (a) Time domain approximation and (b) the power spectrum of the approximation and the data for a lightly filled sample with 3 lb tip mass.

$$
u(t, 0)=0, \quad u(0, x)=\varphi_{0}, \quad \frac{\partial u}{\partial t}(0, x)=0, \quad \varepsilon_{1}(0, x)=0
$$

where, in general, the derivatives of $g_{v}$ in (14) are distributional in the sense described earlier. The parameter identification problem was solved using MATLAB optimization routines. Typical results for the identification problem are shown in Figure 4, where a $3 \mathrm{lb}$ tip mass was used at the bottom of the sample. The identification problem was also run on data obtained from experiments with $2 \mathrm{lb}$ and $1 \mathrm{lb}$ tip mass added to the lightly filled sample. We found a similar good agreement between the data and the model [21]. However, the identified coefficients were not entirely consistent across experiments, although they should describe the same material. This variation is probably caused by the substantially different stress and strain rate ranges involved in the experiments, and suggests that the model should be refined to account for these differences.

We repeated the experiment with a highly filled sample having a $9.29 \mathrm{lb}$ tip mass. In this case our best fit depicted in Figure 5 (a) has deteriorated. Thus we turned to a modification of our model to obtain a better approximation to the force data.

This modification was motivated by our earlier efforts as well as those reported in the literature on similar problems involving hysteretic effects and internal strain models (for details, see the discussions in [21]). We next considered the model using two internal variables $\varepsilon_{1}\left(t ; \tau_{1}\right), \varepsilon_{2}\left(t ; \tau_{2}\right)$ with different decay parameters $\tau_{1}, \tau_{2}$, given by

$$
\begin{aligned}
& \tilde{\rho} \frac{\partial^{2} u}{\partial t^{2}}-A_{c} C_{D} \frac{\partial^{3} u}{\partial t \partial x^{2}}-\frac{\partial}{\partial x}\left(A_{c} g_{e}\left(\frac{\partial u}{\partial x}\right)+A_{c} \varepsilon_{1}+A_{c} \varepsilon_{2}\right)=F(t) \text { in } V^{*} \\
& \dot{\varepsilon}_{1}=-\frac{1}{\tau_{1}} \varepsilon_{1}+\frac{d}{d t} g_{v}\left(\frac{\partial u}{\partial x}, \frac{\partial^{2} u}{\partial t \partial x}\right)
\end{aligned}
$$



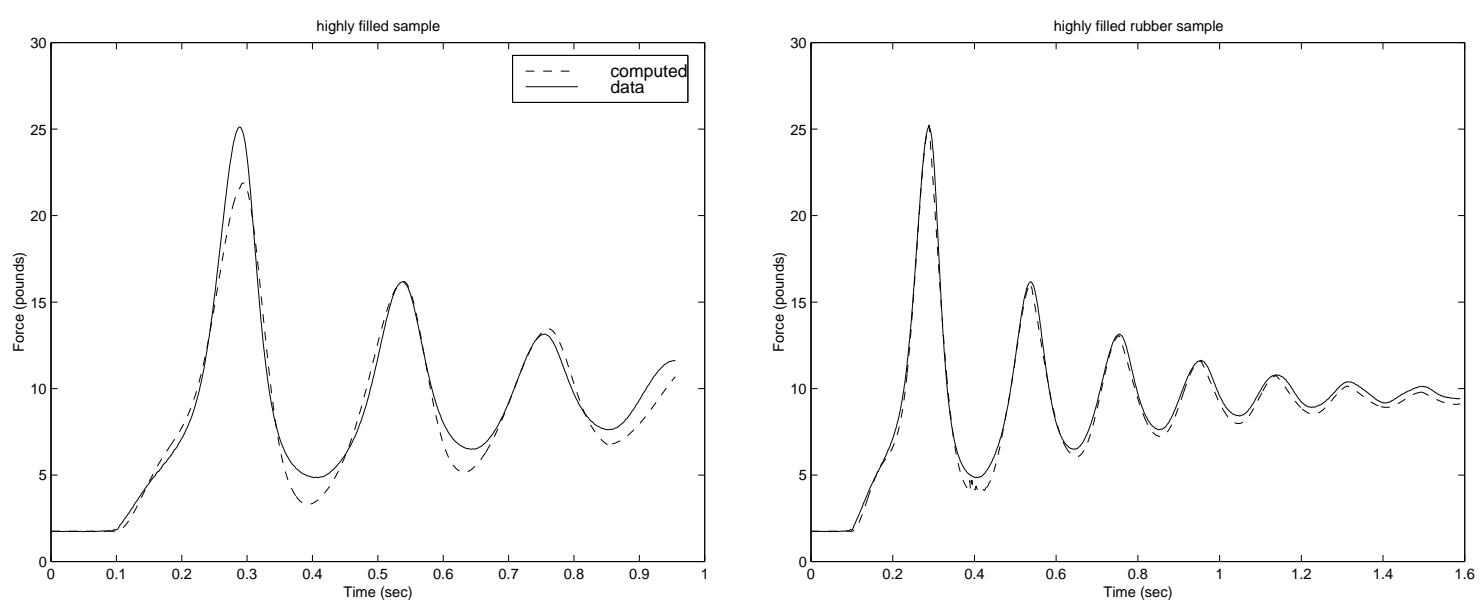

Figure 5: (a) Best approximation for the highly filled sample with one internal variable using data in the time interval $[0,0.95]$ and (b) Best approximation for the highly filled sample with two internal variables on the full time interval. Identification based on data from the time interval $[0,0.95]$.

$$
\begin{aligned}
& \dot{\varepsilon}_{2}=-\frac{1}{\tau_{2}} \varepsilon_{2}+\frac{d}{d t} g_{v}\left(\frac{\partial u}{\partial x}, \frac{\partial^{2} u}{\partial t \partial x}\right) \\
& u(t, 0)=0, \quad u(0, x)=\varphi_{0}, \quad \frac{\partial u}{\partial t}(0, x)=0, \quad \varepsilon_{1}(0, x)=0, \quad \varepsilon_{2}(0, x)=0 .
\end{aligned}
$$

After parameter estimation, this model provided a very satisfactory fit to the data with the relative error between the data and the computed force being $4.1 \%$. This fit, depicted in Figure 5 (b), was obtained by using data only from the time interval [0,0.95], and resulted in a model that accurately simulated the data on the interval $[0,1.6]$.

\subsection{Nonlinear internal variable models}

Our last result for the highly filled sample and the variation in the parameters for the lightly filled rubber rod suggested that we might try to generalize our model to better describe the behavior of highly hysteretic samples. Thus we next considered internal variable models with nonlinear internal dynamics

$$
\begin{aligned}
& \tilde{\rho} \frac{\partial^{2} u}{\partial t^{2}}-A_{c} C_{D} \frac{\partial^{3} u}{\partial t \partial x^{2}}-\frac{\partial}{\partial x}\left(A_{c} g_{e}\left(\frac{\partial u}{\partial x}\right)+A_{c} \varepsilon_{1}\right)=F(t) \text { in } V^{*} \\
& \dot{\varepsilon}_{1}=-g^{\varepsilon}\left(\varepsilon_{1}\right)+\frac{d}{d t} g_{v}\left(\frac{\partial u}{\partial x}, \frac{\partial^{2} u}{\partial t \partial x}\right) \\
& u(t, 0)=0, \quad u(0, x)=\varphi_{0}, \quad \frac{\partial u}{\partial t}(0, x)=0, \quad \varepsilon_{1}(0, x)=0 .
\end{aligned}
$$

In [23] we showed that this system has a unique weak solution. We also note that we in fact generalized the previous existence-uniqueness result in the sense that we no longer require 
monotonicity of the nonlinear functions $g_{e}, g_{v i}, g_{v d}$. Instead, they are assumed to satisfy a local Lipschitz property and other assumptions similar to those used before. We remark that similar techniques were successfully employed to establish existence-uniqueness of weak solutions for linear evolution equations of second order in $t$ in [24] and for semilinear second order evolution equations where the nonlinear term satisfies a global Lipschitz condition in [25]. In [26] we used similar techniques to study a nonlinear beam equation where the nonlinearity satisfies only a local Lipschitz condition.

\subsection{Model development, experimental and numerical results for simple shear}

The previous model development and identification process was also carried out for filled elastomers undergoing simple shear deformation. A dynamic experiment was designed that involved a "double sandwich" fixture with layers of filled rubber at the interfaces (see Figure 6 ). The side bars were fixed, while the middle bar was perturbed by an impulsive hammer hit, and accelerometer and load cell data were collected as in the experiments for extension. For simple shear the model developed and used was

$$
\begin{aligned}
& \rho A_{c} \frac{\partial^{2} u}{\partial t^{2}}=\frac{\partial}{\partial x}\left[A_{c} g_{e}\left(\frac{\partial u}{\partial x}\right)+A_{c} C_{D} \frac{\partial^{2} u}{\partial t \partial x}+A_{c} \varepsilon_{1}\right] \\
& M \frac{\partial^{2} u}{\partial t^{2}}(t, \ell)=-\left.A_{c}\left[g_{e}\left(\frac{\partial u}{\partial x}\right)+C_{D} \frac{\partial^{2} u}{\partial t \partial x}+\varepsilon_{1}\right]\right|_{x=\ell}+f(t)+M g \\
& \frac{d}{d t} \varepsilon_{1}=-\frac{1}{\tau} \varepsilon_{1}+\frac{d}{d t} g_{v}\left(\frac{\partial u}{\partial x}, \frac{\partial^{2} u}{\partial t \partial x}\right) \\
& u(t, 0)=0, \quad u(0, x)=\varphi_{0}, \quad \frac{\partial u}{\partial t}(0, x)=0, \quad \varepsilon_{1}(0, x)=0 .
\end{aligned}
$$

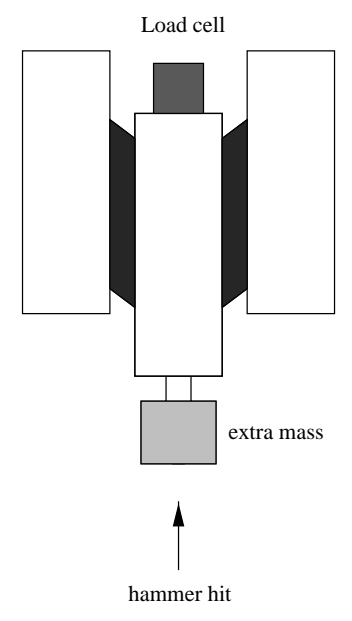

Figure 6: Schematic of the initial shear experimental device. 
In the identification process we used the accelerometer data to approximate the unknown parameters, i.e., we solved the least-square minimization problem

$$
J(q)=\frac{1}{2} \sum_{i=1}^{N}\left|a_{i}-\frac{\partial^{2} u}{\partial t^{2}}\left(\bar{t}_{i}, \ell ; q\right)\right|^{2},
$$

where $a_{i}$ represent the collected data, and $q$ is a vector of unknown coefficients. The experiment was performed with lightly filled and highly filled rubber samples with different amounts of extra mass. In general, we found that the data collected with no extra mass on the sample could be approximated well with the above model even when the viscoelastic response $g_{v}$ is assumed to be linear. This is not surprising, since the maximum strain levels in these type of experiments were below $10 \%$. Our best fit for the highly filled sample with no extra mass is depicted in Figure 7. However, when extra mass was involved to achieve larger deformations and strain levels, the experiment did not provide suitable data, since additional "tilting" modes became excited that could not be accounted for by the above one-dimensional model.

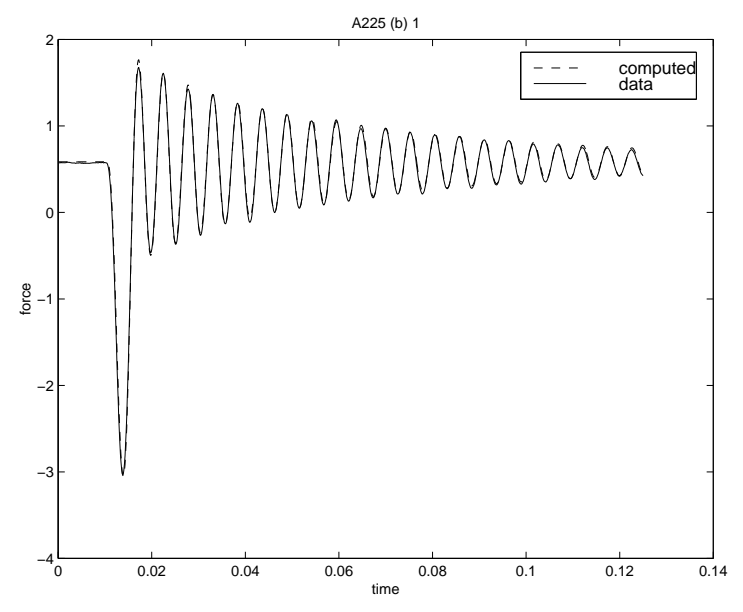

Figure 7: Highly filled rubber sample in shear with no extra mass

The experiment was redesigned to enforce simple shear in the sample with no additional deformations. These new experiments involved four "double sandwich" samples with highly filled rubber at the end of four arms of a fixture. The arms were latched down to achieve a prescribed initial strain (50\%-100\%) in the sample and subsequently released. We again collected accelerometer and load cell data. The identification problem was first performed for the data obtained for $100 \%$ initial strain. We tried different hysteretic stress-strain relationships in our numerical simulations. We note that a linear viscoelastic response function $g_{v}$ was no longer adequate to describe the data in this higher strain regime. Thus, we initially assumed

$$
\sigma=g_{e}\left(\frac{\partial u}{\partial x}\right)+C_{D} \frac{\partial^{2} u}{\partial t \partial x}+\varepsilon_{1}, \quad \dot{\varepsilon}_{1}+\frac{1}{\tau} \varepsilon_{1}=g_{v}\left(\frac{\partial u}{\partial x}\right)
$$


where $g_{e}$ and $g_{v}$ are cubic polynomials and $g_{v}$ does not depend on $\dot{\varepsilon}$, (i.e., $g_{v i}=g_{v d}$ in (9)). The parameters that provided the best fit in this case did not work well for the $70 \%$ and $50 \%$ initial strain experiments. Thus we next used the full nonlinear hysteretic model (i.e., $g_{v i} \neq g_{v d}$ in (9)) to approximate the $100 \%$ initial strain data. The best fit in this case is depicted in Figure 8 (a). We found that the set of parameters identified for this case described the data with $70 \%$ initial strain with very good accuracy (see Figure 8 (b)).
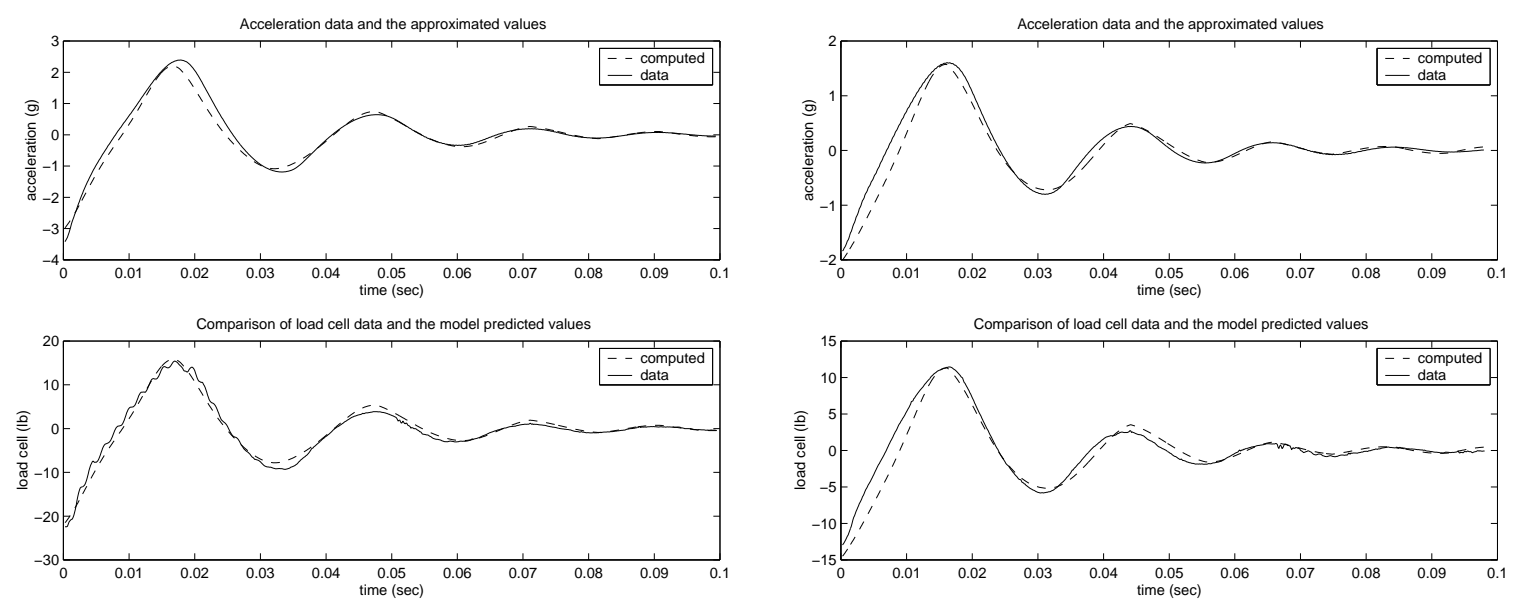

Figure 8: (a) Approximation result for the highly filled sample in shear with $\approx 100 \%$ strain initial condition and (b) The same parameter set simulating the highly filled sample in shear with $\approx 70 \%$ strain initial condition.

\subsection{Molecular based reptation models}

The above models and the nonlinear extensions of Section 2.3 have not, to date, provided insight into the underlying mechanisms for tensile and/or shear deformations in filled rubber. This is not unexpected since the approaches described above are based on pseudophenomenological formulations. More recently $([5,27])$, we have turned to a different approach based on molecular arguments which, as we shall see, lead precisely to the class of models based on a Boltzmann hysteresis formulation. As usual, one begins with force and moment balance and seeks constitutive laws (such as (10)) for the viscoelastic stress term $\sigma_{\text {visco }}$ in

$$
\sigma(t ; \tau)=\sigma_{\text {elast }}(\varepsilon, \dot{\varepsilon})+\sigma_{\text {visco }}\left(\varepsilon_{1}(\cdot)\right)
$$

where $\varepsilon=\frac{\partial u}{\partial x}$ is the infinitesimal strain and $\varepsilon_{1}$ is an "internal strain" variable on which $\sigma_{v i s c o}$ depends in an hysteretic manner. As described above, we found that a reasonable description of the data could be given with the typical stress-strain relationship

$$
\sigma(t)=\tilde{g}_{e}(\varepsilon(t), \dot{\varepsilon}(t))+\int_{0}^{t} \gamma e^{-\frac{t-s}{\tau}} \frac{d}{d s} g_{v}(\varepsilon(s), \dot{\varepsilon}(s)) d s
$$


where $\tau$ is a relaxation parameter, $g_{v}$ is as defined in (9), with cubic polynomials $g_{v i}, g_{v d}$, and $\tilde{g}_{e}=g_{e}\left(\frac{\partial u}{\partial x}\right)+C_{D} \frac{\partial^{2} u}{\partial t \partial x}$ as in (4). We have already observed that this expression is equivalent to

$$
\sigma(t)=\tilde{g}_{e}(\varepsilon(t), \dot{\varepsilon}(t))+\gamma \varepsilon_{1}(t ; \tau)
$$

where, for a given "relaxation parameter" $\tau$, the internal strain $\varepsilon_{1}(t ; \tau)$ satisfies (8). In fact, we found that highly filled rubbers required multiple relaxation times $\tau_{1}, \tau_{2}$ as in (16)-(19) to obtain good model fits to the data. As we shall describe, molecular based formulations, where microscopic relaxation parameters vary across the population of molecules in the material, lead to internal dynamics of the form (8),(9). When combined with a Prohorov metric framework (see $[28,29]$ ) for uncertainty in internal dynamics, these ideas lead to the computational models we have used above. Indeed, the molecular based approach leads to a general class of models with uncertainty or randomness in the stress

$$
\sigma(t, x ; P)=\tilde{g}_{e}(\varepsilon(t, x), \dot{\varepsilon}(t, x))+\gamma \int_{\mathcal{T}} \varepsilon_{1}(t, x ; \tau) d P(\tau),
$$

where $P$ is a probability distribution over the set $\mathcal{T}$ of possible relaxation parameters, and $\varepsilon_{1}(t ; \tau)$ satisfies, for each $\tau \in \mathcal{T}$,

$$
\dot{\varepsilon}_{1}(t, x ; \tau)+\frac{1}{\tau} \varepsilon_{1}(t, x ; \tau)=\dot{\varepsilon}(t, x) h(\varepsilon(t, x)) .
$$

For the reptation model derivation in [5], one begins with the Doi/Edwards [3] stick-slip molecular models as embodied in the continuous tube reptation models of Johnson/Stacer [4] wherein polymer materials such as rubber are postulated to be composed of two types of molecules. In tensile deformations, one denotes by $L(t)$ the length of chemically cross-linked or CC molecules, while $\ell(t)$ denotes the length of physically constrained or PC molecules. To use stick-slip models in continuum simulations of reptation in rubbers, one considers networks of "cells" or boxes of parallel-sided CC boxes and PC boxes with sides of length (principal stretches)

$$
\lambda_{c}=1+\varepsilon=1+\frac{\partial u_{c}}{\partial x}, \quad \lambda_{p}=1+\varepsilon_{1}=1+\frac{\partial u_{p}}{\partial x},
$$

respectively. Here $u_{c}$ denotes the deformations of the $\mathrm{CC}$ box and $u_{p}$ denotes the deformations of the PC box. Using a linear stick-slip assumption as in [4], and strain energy densities based on experiments of Young and Danik (see $[5,27]$ for details), one obtains as a limit of $\mathrm{PC}$ response to step tensile deformations of the $\mathrm{CC}$ molecules, the $\varepsilon, \varepsilon_{1}$ coupled dynamics

$$
\dot{\varepsilon}_{1}+\frac{1}{\tau} \varepsilon_{1}=\dot{\varepsilon} \frac{1+\varepsilon_{1}}{1+\varepsilon} .
$$

However, if one replaces the linear assumption of [27] by a nonlinear stick-slip hypothesis (which is the basis of the work in [5]), one obtains a more general nonlinear, dynamical relationship between $\varepsilon$ and $\varepsilon_{1}$ given by

$$
\dot{\varepsilon}_{1}+\frac{1}{\tau} \varepsilon_{1}=\dot{\varepsilon} f\left(\left(1+\varepsilon_{1}\right) /(1+\varepsilon)\right) .
$$


Expansion and truncation of higher order terms lead to equations of the form

$$
\dot{\varepsilon}_{1}+\frac{1}{\tau} \varepsilon_{1}=\dot{\varepsilon}\left(\alpha_{0}+\alpha_{1} \varepsilon+\alpha_{2} \varepsilon^{2}+\alpha_{3} \varepsilon^{3}\right)
$$

which are of the same form as the internal variable model $(8),(9)$ with $g_{v i}$ and $g_{v d}$ cubic polynomials. For the corresponding contributions to $\sigma$ from the strain energy densities of Young-Danik/ Johnson-Stacer with the nonlinear stick-slip hypothesis, one obtains a contribution to the rate independent strain $g_{v}^{s}$ (after expanding $f$ in a Taylor series and dropping higher order terms) of the form

$$
g_{v}^{s}\left(\varepsilon, \varepsilon_{1}\right)=g_{c u b i c}(\varepsilon)+\gamma_{1} \varepsilon_{1}
$$

where $\varepsilon_{1}$ is as before (i.e., the internal strain satisfying (21)). Thus, the total stress-strain relationship can be written in the form (20). If the measure $\mathrm{P}$ of $(20)$ has atoms at $\tau_{1}$ and $\tau_{2}$, (i.e., the measure is composed of Dirac measures concentrated at $\tau_{1}$ and $\tau_{2}$ ), then the constitutive law leads precisely to the model

$$
\sigma(t, x ; P)=\tilde{g}_{e}(\varepsilon(t, x), \dot{\varepsilon}(t, x))+\gamma_{1} \varepsilon_{1}\left(t, x ; \tau_{1}\right)+\gamma_{2} \varepsilon_{2}\left(t, x ; \tau_{2}\right),
$$

which was used in the data fit of Section 2.2 with model (16)-(19).

\section{Concluding Remarks}

In the above note we outlined our progress to date in the development of nonlinear dynamic models for inactive filled elastomers. Substantial experimental validation for our approach is provided both in the quasi-static and dynamic cases in uniaxial tension and in the dynamic case in simple shear. Current efforts involve refinements to these models and a comparison with newly developed molecular based models $[5,27]$ that emphasize uncertainty or randomness across populations of molecules in a heterogeneous material.

\section{Acknowledgments}

This research was supported in part by the U.S. Air Force Office of Scientific Research under grants AFOSR F49620-01-1-0026 (HTB and NGM) and AFOSR F49620-03-1-0185 (GAP).

\section{References}

[1] H.T. Banks, R.C. Smith and Y. Wang, Smart Material Structures: Modeling, Estimation and Control, Masson, Paris and John Wiley and Sons, Chichester, 1996.

[2] M.V. Gandhi and B.S. Thompson, Smart Materials and Structures, Chapman and Hall, London, 1992.

[3] M. Doi and M. Edwards, The Theory of Polymer Dynamics, Oxford, New York, 1986. 
[4] A.R. Johnson and R.G. Stacer, Rubber viscoelasticity using the physically constrained systems' stretches as internal variables, Rubber Chem. Tech. 66, 567-577 (1993).

[5] H.T. Banks, N.G. Medhin and G.A. Pinter, Nonlinear reptation in molecular based hysteresis models for polymers, in progress.

[6] R.M. Christensen, Theory of Viscoelasticity: An Introduction, 2nd ed., Academic Press, New York, 1982.

[7] W.N. Findley, J.S. Lai and K. Onaran, Creep and Relaxation of Nonlinear Viscoelastic Materials, North-Holland Series in Applied Mathematics and Mechanics, North-Holland Publishing Company, Amsterdam, 1976.

[8] J.D. Ferry, Viscoelastic Properties of Polymers, John Wiley \& Sons, Inc., New York, 1980.

[9] R.W. Ogden, Non-linear Elastic Deformations, Ellis Horwood Limited, Chichester, 1984.

[10] R.S. Rivlin, Large elastic deformations of isotropic materials, I, II, III., Phil. Trans. Roy. Soc. A 240, 459-525 (1948).

[11] R.A. Schapery, On the characterization of nonlinear viscoelastic materials, Polymer Engineering and Science 9, 295-310 (1969).

[12] H.T. Banks and N. Lybeck, Modeling methodology for elastomer dynamics, in Systems and Control in the 21st Century, (C. Byrnes, et.al., eds.), Birkhäuser, Boston, 1996, 37-50.

[13] H.T. Banks, N.J. Lybeck, M.J. Gaitens, B.C. Muñoz and L.C. Yanyo, Computational methods for estimation in the modeling of nonlinear elastomers, CRSC-TR95-40, NCSU, December 1995; Kybernetika 32, 526-542 (1996).

[14] H.T. Banks, D.S. Gilliam and V.I. Shubov, Global solvability for damped abstract nonlinear hyperbolic systems, Differential and Integral Equations 10, 309-332 (1997).

[15] H.T. Banks and G.A. Pinter, Approximation results for parameter estimation in nonlinear elastomers, CRSC-TR96-34, NCSU, December 1996; Control and Estimation of Distributed Parameter Systems, (F. Kappel, et.al.,eds.), Birkhäuser, Boston, 1998, 1-13.

[16] H.T. Banks and G.A. Pinter, Damping: hysteretic damping and models, CRSC-TR9936, NCSU, December 1999; Encyclopedia of Vibrations, Academic Press, London, 2001, 658-664.

[17] G.A. Lesieutre, Modeling frequency-dependent longitudinal dynamic behavior of linear viscoelastic long fiber components, J. Composite Materials 28, 1770-1782 (1994). 
[18] G.A. Lesieutre and K. Govindswamy, Finite element modeling of frequency-dependent and temperature-dependent dynamic behavior of viscoelastic materials in simple shear, Int. J. Solids Structures 33, 419-432 (1996).

[19] A.R. Johnson, C.J. Quigley and J.L. Mead, Large strain viscoelastic constitutive models for rubber, part I: Formulations, Rubber Chemistry Technology 67, 904-917 (1994).

[20] H.T. Banks, G.A. Pintér, and L.K. Potter, Existence of unique weak solutions to a dynamical system for nonlinear elastomers with hysteresis, CRSC-TR98-43, NCSU, November 1998; Differential and Integral Equations 13, 1001-1024 (2000).

[21] H.T. Banks, G.A. Pintér, L.K. Potter, M.J. Gaitens and L.C. Yanyo, Modeling of nonlinear hysteresis in elastomers under uniaxial tension, J. Intelligent Material Systems and Structures 10, 116-134 (1999).

[22] Y. Zhang, Mathematical formulation of vibrations of a composite curved beam structure: Aluminum core material with viscoelastic layers, constraining layers and piezoceramic patches, Ph.D. Thesis, N.C. State University, May 1997.

[23] A.C. Ackleh, H.T. Banks and G.A. Pinter, Well-posedness results for models of elastomers, J. Math. Analysis and Applications 268, 440-456 (2002).

[24] R. Dautray and J.L. Lions, Mathematical Analysis and Numerical Methods for Science and Technology, Volume 5, Evolution Problems I, Springer, New York, 2000.

[25] J. Ha and S. Nakagiri, Existence and regularity of weak solutions for semilinear second order evolution equations, Funkcialaj Ekvacioj 41, 1-24 (1998).

[26] A.C. Ackleh, H.T. Banks and G.A. Pinter, On a nonlinear beam equation, Applied Math. Letters 15, 381-387 (2002).

[27] H.T. Banks and N.G. Medhin, A molecular based dynamic model for viscoelastic responses of rubber in tensile deformations, CRSC-TR00-27, NCSU, October, 2000; Communications on Applied Nonlinear Analysis 8, 1-18 (2001).

[28] H.T. Banks and K. Bihari, Modeling and estimating uncertainty in parameter estimation, CRSC-TR99-40, NCSU, December 1999; Inverse Problems 17, 1-17 (2001).

[29] H.T. Banks, D. Bortz, G.A. Pinter and L.K. Potter, Modeling and imaging techniques with potential application in bioterrorism, CRSC-TR03-02, NCSU, January 2003; Chapter 6 in Bioterrorism: Mathematical Modeling Applications in Homeland Security, (H.T. Banks and C. Castillo-Chavez, eds.), Frontiers in Applied Mathematics, Vol.28, SIAM, Philadelphia, 2003, 129-154. 